\title{
Structure, Spectroscopic and Quantum Chemical Investigations of 4-Amino-2-Methyl-8-(Trifluoromethyl)Quinoline
}

\author{
*T. Raci Sertbakan \\ Department of Physics, Faculty of Art and Science, Ahi Evran University, 40100, Kırsehir, Turkey, \\ trsertbakan@ahievran.edu.tr \\ *Corresponding Author
}

Received: 18 June 2017

Accepted: 31 October 2017

DOI: $10.18466 /$ cbayarfbe. 339858

\begin{abstract}
This work deals with the spectroscopic properties (FT-IR, FT-Raman and NMR), structural and some electronic properties as well as theoretical calculations of 4-amino-2-methyl-8-(trifluoromethyl) quinoline (AMTQ) molecule. The vibrational, structural and some electronic properties observations of the AMTQ were reported, which is investigated using some spectral methods and DFT calculations. FT-IR and FT-Raman spectra were obtained for AMTQ at room temperature in the region $4000 \mathrm{~cm}^{-1}-400 \mathrm{~cm}^{-1}$ and $3500-50 \mathrm{~cm}^{-1}$, respectively. In the DFT calculations, the B3LYP functional with cc-pVDZ, cc-pVTZ and cc-pVQZ basis sets was applied to carry out the quantum mechanical calculations of the spectroscopic, structural and some electronic properties of AMTQ. FT-IR and FT-Raman spectra were interpreted with the by using of normal coordinate analysis based on scaled quantum mechanical force field. The present work expands our understanding of the both the vibrational and structural properties as well as some electronic properties of the AMTQ by means of the theoretical and experimental methods.
\end{abstract}

Keywords: 4-amino-2-methyl-8-(trifluoromethyl)quinoline, FT-IR and FT-Raman spectra, NMR spectra, DFT, Electronic properties

\section{Introduction}

Quinoline and its derivatives possess an aromatic heterocyclic unit of benzene connected to a pyridine ring. It is a heterocyclic aromatic organic compound with the chemical formula $\mathrm{C}_{9} \mathrm{H}_{7} \mathrm{~N}$. It is a colorless hygroscopic liquid with a strong odor. Aged samples, especially if exposed to light, become yellow and later brown. Quinoline is only slightly soluble in cold water but dissolves readily in hot water and most organic solvents. Quinoline itself has few applications, but many of its derivatives are useful in diverse applications [1]

In addition to the medicinal and therapeutic applications of quinoline derivatives, these compounds have also shown technological properties. Because, it has the unique structural and electronic properties. Quinoline and its derivatives presents the electronic delocalization and material nonlinear optical properties in the manifestation of fluorescence effects [2]. They were given the important developments in the field of organic light emitting diodes [3-5].

Vibrational spectroscopy in combination with computational chemistry has been used systematically over the past decade to elucidate the spectroscopic, structures and some electronic properties of molecule. Various researchers [6-11] used simulation methods such as DFT, HF simulations to explained molecular structure and vibrational spectra. Such study supports the experimental data in interpreting the calculations results of the structural and spectroscopic properties of the isolated molecule. Erdogdu et al. [12-15] studied vibrational properties of the several molecules. Experimental results were favorable agreed with the results obtained by simulations.

Quinoline derivatives has been applied the characterize by Raman spectroscopy [16,17,20-22]. Especially, aminoquinolines have chromophoric groups and highly symmetric molecular structures. Therefore, they give rise to intense Raman signals $[16,18,19,23,24]$. The vibrational spectra of the some substituted quinolones have been experimentally recorded and theoretically analyzed [21,25-27]. It is reported that the solid state structure for 4,7- dichloroquinoline has been described [28]. In that work, Authors showed the two molecules with the quinolone asymmetric unit. That compounds have the crystallizes in a monoclinic system with $\mathrm{P}_{21 / n}$ space group. Some quinolone derivative such as 4,7dichloroquinoline and quinolin-8-ol, and 4-azido-7- 
chloroquinoline molecules have been characterized by the Raman and infrared spectra. In that work, the 4azido-7-chloroquinoline compound was synthesized from 4,7-dichloroquinoline according to the method described by Pereira et al. [29]. The molecular structure and vibrational spectra of these molecules have been calculated by DFT (B3LYP with 6-311++G(d,p) ).

\section{Theoretical and Experimental Details}

Density functional theory (DFT) computations for the geometric optimization and frequency calculation were performed using Gaussian 09 program [30]. The calculations employed the B3LYP exchange-correlation functional, which combines the hybrid exchange functional of Becke with the gradient-correlation functional of Lee et al. and the cc-pVDZ, cc-pVTZ and cc-pVQZ basis set. Calculations were based on the isolated molecule model, so the environmental effects were not considered. The optimal geometry was determined by minimizing the energy with respect to all geometrical parameters without imposing molecular symmetry constraints. In the optimized structure, imaginary frequency modes were absent which provided a true minimum picture of the potential energy surface.

Following the geometry optimizations with B3LYP method, the optimized structural parameters used in the vibrational wavenumber calculation at DFT level to characterize all stationary points while minima. The potential energy distribution (PED) was calculated by using the scaled quantum mechanics (SQM) program [31] and the fundamental vibrational modes were characterized by their PED values. In addition, the frontier molecular orbital analysis and Molecular electrostatic potential (MEP) were performed.

AMTQ was purchased from Aldrich and used without further purification. Infrared spectra of the samples were recorded between for the $400-4000 \mathrm{~cm}^{-1}$ on a Mattson 1000 FTIR spectrometer which was calibrated using polystyrene bands. The samples were prepared as a $\mathrm{KBr}$ disc. FT-Raman spectrum of AMTQ has been recorded in the frequency region $50-3500 \mathrm{~cm}^{-1}$ on a Thermo Scientific DXR Raman Microscope with Nd: $\mathrm{YVO}_{4}$ DPSS laser operating with $532 \mathrm{~nm}$ excitation. The sample was prepared as a $\mathrm{KBr}$ disc. The ${ }^{13} \mathrm{C}$ NMR spectra were recorded in chloroform solutions and all signals are referenced to TMS on a Bruker Ultrashield FT-NMR Spectrometer. All NMR spectra were measured at room temperature.

\section{Molecular structure}

The optimized structure of conformer of AMTQ was shown in Figure 1 which has the atom numbering scheme adopted in this study. The optimized structural parameters such as dihedral angles, bond angles and bond lengths of the AMTQ were given in Table 1.

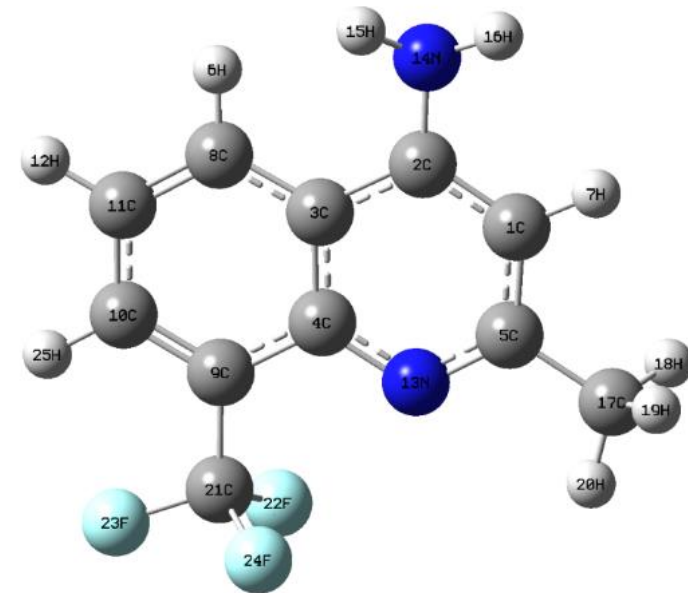

Figure 1. Molecular structure and atomic numbering of the 4-amino-2-methyl-8-(trifluoromethyl)quinoline molecule.

\section{Assignment of Vibrational Spectra}

In order to assist in the assignment of the vibrational bands, predicted infrared and Raman spectra obtained by using DFT approximation. The AMTQ molecule consists of 25 atoms. So, it has 69 normal vibrational modes. Since it belongs to the Cs point group, these normal modes are distributed with 45 in-plane and 24 out-ofplane vibrations. The experimental and calculated infrared and Raman spectra for AMTQ are given in Figures 2 and 3 . The corresponding data are gathered in Table 2 .

The NH stretching vibrations give rise to bands at 3500$3300 \mathrm{~cm}^{-1}$ [32]. According to Roeges, the $\mathrm{N}-\mathrm{H}$ stretching vibration appears strongly and broadly in the region $3390 \pm 60 \mathrm{~cm}^{-1}$ [33]. For the title compound $\mathrm{N}-\mathrm{H}$ stretching modes are assigned at 3383 and $3494 \mathrm{~cm}^{-1}$ theoretically (cc-pVQZ) and a strong band is observed in the IR spectrum at $3383 \mathrm{~cm}^{-1}$ (FT-IR) $3388 \mathrm{~cm}^{-1}$ (FT-Raman). It is well-known that the amino group presents a characteristic band in the $1500-1600 \mathrm{~cm}^{-1}$ range of the IR spectrum, originated by the $\mathrm{NH}_{2}$ deformation mode $\left(\delta_{\mathrm{NH} 2}\right)$. The $\mathrm{NH}_{2}$ deformation band of the title compound detected at $1592 \mathrm{~cm}^{-1}$ (FT-IR) and $1594 \mathrm{~cm}^{-1}$ (FT-Raman). $1585 \mathrm{~cm}^{-1}$ peak predicted as the $\mathrm{NH}_{2}$ deformation band by means of B3LYP/cc-pVQZ level of theory.

The $\mathrm{C}-\mathrm{H}$ stretching vibrations of the aromatic structure appear in the region $3200-3000 \mathrm{~cm}^{-1}$. It's characteristic region for the identification of the $\mathrm{C}-\mathrm{H}$ stretching vibrations [6-11]. The ring, which is coordinated trifluoromethyl group, has the three $\mathrm{CH}$ stretching vibrations. $3072 \mathrm{~cm}^{-1}, 3091 \mathrm{~cm}^{-1}$ and $3114 \mathrm{~cm}^{-1}$ peaks were predicted the $\mathrm{CH}$ stretching vibrations by the B3LYP/cc-pVQZ calculation. According to the PED results, PED contributions were almost calculated as pure modes. In the FT-Raman spectra, $\mathrm{CH}$ stretching 
vibrations of this ring were observed at $3072 \mathrm{~cm}^{-1}$ and $3099 \mathrm{~cm}^{-1}$.

Experimentally, the in-plane $\mathrm{CH}$ bending modes were measured at $1517 / 1525 \mathrm{~cm}^{-1}$ (IR/Raman), $1442 / 1441 \mathrm{~cm}^{-}$ ${ }^{1}$ (IR/Raman) and -/1309 (-/Raman) $\mathrm{cm}^{-1},-/ 1259 \mathrm{~cm}^{-1}$ (-/Raman) 1228/1228 cm-1 (IR/Raman), $1189 / 1182 \mathrm{~cm}^{-1}$ (IR/Raman) and 1143/1144 $\mathrm{cm}^{-1}$ (IR/Raman), whereas computed in-plane $\mathrm{CH}$ bending modes were found at $1501 \mathrm{~cm}^{-1}, 1441 \mathrm{~cm}^{-1}, 1320 \mathrm{~cm}^{-1}, 1252 \mathrm{~cm}^{-1}, 1209 \mathrm{~cm}^{-1}$, $1170 \mathrm{~cm}^{-1}$ and $1161 \mathrm{~cm}^{-1}$ by DFT (B3LYP/cc-pVQZ) calculation. Additionally, the out of plane bending $\mathrm{CH}$ modes were observed at $842(836) \mathrm{cm}^{-1}, 825 \mathrm{~cm}^{-1}$ and 771 (762) $\mathrm{cm}^{-1}$ in the FT-IR (FT-Raman) spectra.

The identification of $\mathrm{C}-\mathrm{N}$ vibration is a very difficult task, since the mixing of several bands is possible in this region. However, with the help of theoretical calculation (DFT), the C-N stretching vibrations are calculated Several bands observed at $13751077 \mathrm{~cm}^{-1}$ in the FTRaman spectrum of the studied compound, with counterpart at $1076 \mathrm{~cm}^{-1}$ in the infrared spectrum are assigned to the $\mathrm{C}-\mathrm{N}$ stretching vibrations. The IR band observed at $1569 \mathrm{~cm}^{-1}, 1619 \mathrm{~cm}^{-1}$ and $1638 \mathrm{~cm}^{-1}$ assigned to the $\mathrm{CC}$ stretching vibrations. Their corresponding counterpart detected at $1572 \mathrm{~cm}^{-1}, 1621 \mathrm{~cm}^{-1}$ and $1637 \mathrm{~cm}^{-1}$ in the FT-Raman spectra. These vibrations calculated at $1557 \mathrm{~cm}^{-1}, 1615 \mathrm{~cm}^{-1}$ and $1603 \mathrm{~cm}^{-1}$ by means of B3LYP/cc-pVQZ level of theory. PED contribution of these vibrations predicted as mixed with $\mathrm{CCC}, \mathrm{CCH}$ bending vibrations.

The C-H stretching frequencies of methyl group appear just below $3000 \mathrm{~cm}^{-1}$ [34,35]. The title molecule possesses methyl $\left(-\mathrm{CH}_{3}\right)$ group. The strong and distinct C-H stretching band at 2924/2925 $\mathrm{cm}^{-1}$ ( FT-IR / FTRaman ) and 2956/2964 $\mathrm{cm}^{-1}$ ( FT-IR / FT-Raman ) are in agreement with the theoretical values of $2933 \mathrm{~cm}^{-1}$ (mode no: 61) and $2979 \mathrm{~cm}^{-1}$ (mode no:62) by B3LYP/cc-pVQZ method and are well supported by the PED values. The asymmetric and symmetric bending vibrations of methyl groups normally appear in the around $1450 \mathrm{~cm}^{-1}$ and around $1350 \mathrm{~cm}^{-1}$ region, respectively $[34,35]$. In the present study, asymmetric $\mathrm{CH}_{3}$ bending vibration (mode no 27) was observed at $1434 \mathrm{~cm}^{-1}$ in the FT-Raman spectrum as very week band and its corresponding counterpart couldn't be detected in the FT-IR spectrum. Asymmetric $\mathrm{CH}_{3}$ bending mode of the methyl group was also predicted at $1423 \mathrm{~cm}^{-1}$ at the B3LYP with cc-pVQZ level of theory. $1421 \mathrm{~cm}^{-1}$ (FT-IR and FT-Raman) peak was assigned to the asymmetric bending vibration of the $\mathrm{CH}_{3}$ group. Symmetric $\mathrm{CH} 3$ peak was calculated at $1333 \mathrm{~cm}^{-1}$ at the B3LYP/cc-pVQZ level of theory. This vibration was measured at $1332 \mathrm{~cm}^{-}$ ${ }^{1}$ in the FT-IR spectra.

The band at $1117 \mathrm{~cm}^{-1}$ in the FT-IR spectrum was appointed as C-F stretching vibration. This mode was predicted $1112 \mathrm{~cm}^{-1}$. According to the calculations $1140 \mathrm{~cm}^{-1}$ and $717 \mathrm{~cm}^{-1}$ are assigned to the symmetric $\mathrm{C}-\mathrm{F}_{3}$ stretching modes, while the $1132 \mathrm{~cm}^{-1}$ vibration is the asymmetric $\mathrm{C}-\mathrm{F}_{3}$ stretching mode. In the predicted spectra, out-of plane F-C-F bending vibration is appointed at $656 \mathrm{~cm}^{-1}$ with $31 \%$ contributions of PED.

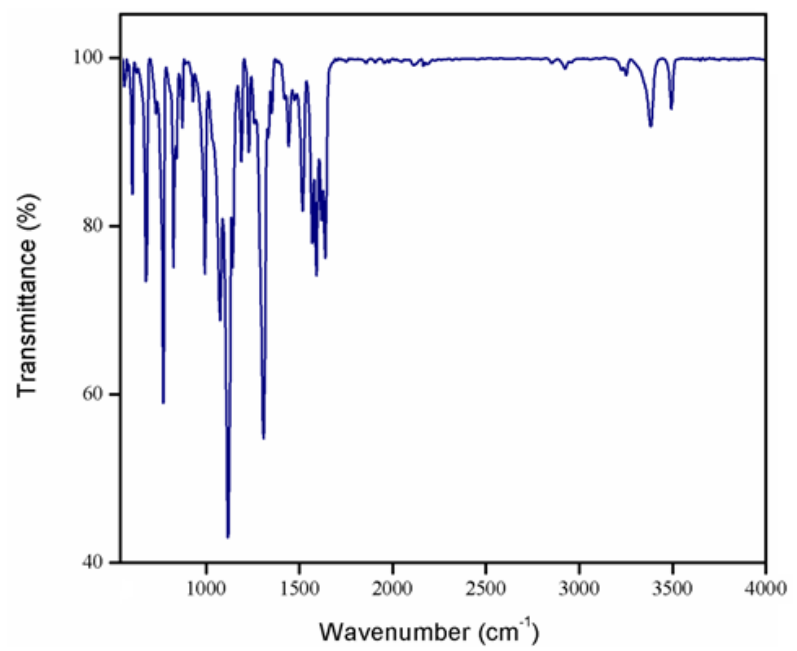

Figure 2. Experimental Infrared spectra of 4-amino-2methyl-8-(trifluoromethyl)quinoline molecule

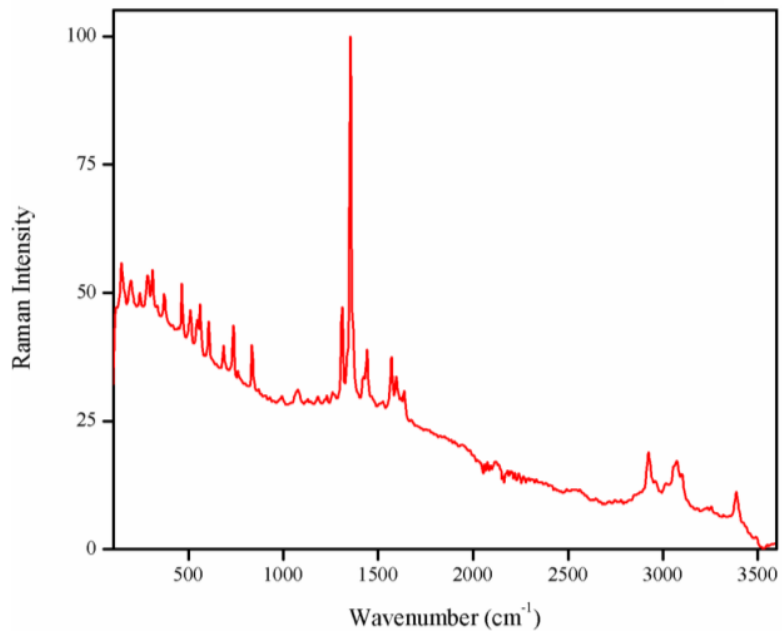

Figure 3. Experimental Raman spectra of 4-amino-2methyl-8-(trifluoromethyl)quinoline molecule 
Table 1. Optimized geometric parameters of 4-amino-2-methyl-8-(trifluoromethyl)quinoline

\begin{tabular}{|c|c|c|c|c|c|c|c|c|c|c|c|}
\hline $\begin{array}{c}\text { Bond } \\
\text { Lengths (Å) }\end{array}$ & cc-pVDZ & cc-pVTZ & cc-pVQZ & $\begin{array}{c}\text { Bond } \\
\left.\text { Angels ( }{ }^{\circ}\right)\end{array}$ & cc-pVDZ & cc-pVTZ & cc-pVQZ & $\begin{array}{c}\text { Dihedral Angels } \\
\left({ }^{\circ}\right)\end{array}$ & cc-pVDZ & cc-pVTZ & cc-pVQZ \\
\hline $\mathrm{C}_{1}-\mathrm{C}_{2}$ & 1.387 & 1.387 & 1.378 & $\mathrm{C}_{2}-\mathrm{C}_{1}-\mathrm{C}_{5}$ & 120.3 & 120.3 & 120.4 & $\mathrm{C}_{5}-\mathrm{C}_{1}-\mathrm{C}_{2}-\mathrm{C}_{3}$ & -0.999 & 1.013 & 0.674 \\
\hline $\mathrm{C}_{1}-\mathrm{C}_{5}$ & 1.417 & 1.417 & 1.409 & $\mathrm{C}_{2}-\mathrm{C}_{1}-\mathrm{H}_{7}$ & 119.9 & 119.9 & 120.0 & $\mathrm{C}_{5}-\mathrm{C}_{1}-\mathrm{C}_{2}-\mathrm{N}_{14}$ & -178.2 & 178.3 & 178.2 \\
\hline $\mathrm{C}_{1}-\mathrm{H}_{7}$ & 1.093 & 1.093 & 1.082 & $\mathrm{C}_{5}-\mathrm{C}_{1}-\mathrm{H}_{7}$ & 119.6 & 119.6 & 119.5 & $\mathrm{H}_{7}-\mathrm{C}_{1}-\mathrm{C}_{2}-\mathrm{C}_{3}$ & 179.8 & -179.8 & -179.8 \\
\hline $\mathrm{C}_{2}-\mathrm{C}_{3}$ & 1.440 & 1.440 & 1.432 & $\mathrm{C}_{1}-\mathrm{C}_{2}-\mathrm{C}_{3}$ & 117.7 & 117.7 & 117.8 & $\mathrm{H}_{7}-\mathrm{C}_{1}-\mathrm{C}_{2}-\mathrm{N}_{14}$ & 2.531 & -2.525 & -2.262 \\
\hline $\mathrm{C}_{2}-\mathrm{N}_{14}$ & 1.384 & 1.384 & 1.377 & $\mathrm{C}_{1}-\mathrm{C}_{2}-\mathrm{N}_{14}$ & 121.7 & 121.8 & 121.3 & $\mathrm{C}_{2}-\mathrm{C}_{1}-\mathrm{C}_{5}-\mathrm{N}_{13}$ & -0.727 & 0.736 & 0.611 \\
\hline $\mathrm{C}_{3}-\mathrm{C}_{4}$ & 1.432 & 1.432 & 1.423 & $\mathrm{C}_{3}-\mathrm{C}_{2}-\mathrm{N}_{14}$ & 120.4 & 120.4 & 120.7 & $\mathrm{C}_{2}-\mathrm{C}_{1}-\mathrm{C}_{5}-\mathrm{C}_{17}$ & 179.9 & -179.9 & -179.5 \\
\hline $\mathrm{C}_{3}-\mathrm{C}_{8}$ & 1.418 & 1.418 & 1.411 & $\mathrm{C}_{2}-\mathrm{C}_{3}-\mathrm{C}_{4}$ & 117.1 & 117.1 & 117.1 & $\mathrm{H}_{7}-\mathrm{C}_{1}-\mathrm{C}_{5}-\mathrm{N}_{13}$ & 178.4 & -178.4 & -178.8 \\
\hline $\mathrm{C}_{4}-\mathrm{C}_{9}$ & 1.432 & 1.432 & 1.426 & $\mathrm{C}_{2}-\mathrm{C}_{3}-\mathrm{C}_{8}$ & 123.1 & 123.1 & 123.2 & $\mathrm{H}_{7}-\mathrm{C}_{1}-\mathrm{C}_{5}-\mathrm{C}_{17}$ & -0.887 & 0.897 & 0.957 \\
\hline $\mathrm{C}_{4}-\mathrm{N}_{13}$ & 1.362 & 1.362 & 1.355 & $\mathrm{C}_{4}-\mathrm{C}_{3}-\mathrm{C}_{8}$ & 119.6 & 119.6 & 119.6 & $\mathrm{C}_{1}-\mathrm{C}_{2}-\mathrm{C}_{3}-\mathrm{C}_{4}$ & 2.055 & -2.081 & -1.530 \\
\hline $\mathrm{C}_{5}-\mathrm{N}_{13}$ & 1.324 & 1.324 & 1.316 & $\mathrm{C}_{3}-\mathrm{C}_{4}-\mathrm{C}_{9}$ & 118.0 & 118.0 & 118.0 & $\mathrm{C}_{1}-\mathrm{C}_{2}-\mathrm{C}_{3}-\mathrm{C}_{8}$ & -177.2 & 177.2 & 178.1 \\
\hline $\mathrm{C}_{5}-\mathrm{C}_{17}$ & 1.508 & 1.508 & 1.504 & $\mathrm{C}_{3}-\mathrm{C}_{4}-\mathrm{N}_{13}$ & 123.5 & 123.5 & 123.1 & $\mathrm{~N}_{14}-\mathrm{C}_{2}-\mathrm{C}_{3}-\mathrm{C}_{4}$ & 179.3 & -179.4 & -179.1 \\
\hline $\mathrm{H}_{6}-\mathrm{C}_{8}$ & 1.092 & 1.092 & 1.081 & $\mathrm{C}_{9}-\mathrm{C}_{4}-\mathrm{N}_{13}$ & 118.3 & 118.3 & 118.7 & $\mathrm{~N}_{14}-\mathrm{C}_{2}-\mathrm{C}_{3}-\mathrm{C}_{8}$ & 0.093 & -0.121 & 0.600 \\
\hline $\mathrm{C}_{8}-\mathrm{C}_{11}$ & 1.380 & 1.380 & 1.371 & $\mathrm{C}_{1}-\mathrm{C}_{5}-\mathrm{N}_{13}$ & 123.2 & 123.2 & 122.9 & $\mathrm{C}_{1}-\mathrm{C}_{2}-\mathrm{N}_{14}-\mathrm{H}_{15}$ & -149.4 & 149.4 & 155.1 \\
\hline $\mathrm{C}_{9}-\mathrm{C}_{10}$ & 1.382 & 1.382 & 1.373 & $\mathrm{C}_{1}-\mathrm{C}_{5}-\mathrm{C}_{17}$ & 119.6 & 119.6 & 119.6 & $\mathrm{C}_{1}-\mathrm{C}_{2}-\mathrm{N}_{14}-\mathrm{H}_{16}$ & -15.91 & 15.89 & 15.32 \\
\hline $\mathrm{C}_{9}-\mathrm{C}_{21}$ & 1.512 & 1.512 & 1.512 & $\mathrm{~N}_{13}-\mathrm{C}_{5}-\mathrm{C}_{17}$ & 117.0 & 117.0 & 117.3 & $\mathrm{C}_{3}-\mathrm{C}_{2}-\mathrm{N}_{14}-\mathrm{H}_{15}$ & 33.31 & -33.28 & -27.30 \\
\hline $\mathrm{C}_{10}-\mathrm{C}_{11}$ & 1.412 & 1.412 & 1.404 & $\mathrm{C}_{3}-\mathrm{C}_{8}-\mathrm{H}_{6}$ & 119.9 & 119.9 & 120.2 & $\mathrm{C}_{3}-\mathrm{C}_{2}-\mathrm{N}_{14}-\mathrm{H}_{16}$ & 166.8 & -166.8 & -167.1 \\
\hline $\mathrm{C}_{10}-\mathrm{H}_{25}$ & 1.089 & 1.090 & 1.079 & $\mathrm{C}_{3}-\mathrm{C}_{8}-\mathrm{C}_{11}$ & 121.7 & 120.7 & 120.8 & $\mathrm{C}_{2}-\mathrm{C}_{3}-\mathrm{C}_{4}-\mathrm{C}_{9}$ & 178.7 & -178.6 & -178.9 \\
\hline $\mathrm{C}_{11}-\mathrm{H}_{12}$ & 1.091 & 1.091 & 1.080 & $\mathrm{H}_{6}-\mathrm{C}_{8}-\mathrm{C}_{11}$ & 119.1 & 119.1 & 118.8 & $\mathrm{C}_{2}-\mathrm{C}_{3}-\mathrm{C}_{4}-\mathrm{N}_{13}$ & -1.629 & 1.647 & 1.283 \\
\hline $\mathrm{N}_{14}-\mathrm{H}_{15}$ & 1.014 & 1.014 & 1.004 & $\mathrm{C}_{4}-\mathrm{C}_{9}-\mathrm{C}_{10}$ & 120. & 120.6 & 120.6 & $\mathrm{C}_{8}-\mathrm{C}_{3}-\mathrm{C}_{4}-\mathrm{C}_{9}$ & -1.982 & 1.995 & 1.333 \\
\hline $\mathrm{N}_{14}-\mathrm{H}_{16}$ & 1.015 & 1.015 & 1.005 & $\mathrm{C}_{4}-\mathrm{C}_{9}-\mathrm{C}_{21}$ & 119.7 & 119.7 & 119.8 & $\mathrm{C}_{8}-\mathrm{C}_{3}-\mathrm{C}_{4}-\mathrm{N}_{13}$ & 177.6 & -177.6 & -178.4 \\
\hline $\mathrm{C}_{17}-\mathrm{H}_{18}$ & 1.103 & 1.103 & 1.091 & $\mathrm{C}_{10}-\mathrm{C}_{9}-\mathrm{C}_{21}$ & 119.5 & 119.5 & 119.0 & $\mathrm{C}_{2}-\mathrm{C}_{3}-\mathrm{C}_{8}-\mathrm{H}_{6}$ & 2.444 & -2.459 & -2.132 \\
\hline $\mathrm{C}_{17}-\mathrm{H}_{19}$ & 1.103 & 1.103 & 1.092 & $\mathrm{C}_{9}-\mathrm{C}_{10}-\mathrm{C}_{11}$ & 120.7 & 120.7 & 120.8 & $\mathrm{C}_{2}-\mathrm{C}_{3}-\mathrm{C}_{8}-\mathrm{C}_{11}$ & -179.0 & 179.0 & 179.2 \\
\hline $\mathrm{C}_{17}-\mathrm{H}_{20}$ & 1.097 & 1.097 & 1.086 & $\mathrm{C}_{9}-\mathrm{C}_{10}-\mathrm{H}_{25}$ & 119.3 & 119.3 & 119.6 & $\mathrm{C}_{4}-\mathrm{C}_{3}-\mathrm{C}_{8}-\mathrm{H}_{6}$ & -176.8 & 176.8 & 177.5 \\
\hline $\mathrm{C}_{21}-\mathrm{F}_{22}$ & 1.348 & 1.349 & 1.345 & $\mathrm{C}_{11}-\mathrm{C}_{10}-\mathrm{H}_{25}$ & 119.8 & 119.8 & 119.5 & $\mathrm{C}_{4}-\mathrm{C}_{3}-\mathrm{C}_{8}-\mathrm{C}_{11}$ & 1.647 & -1.656 & -1.078 \\
\hline $\mathrm{C}_{21}-\mathrm{F}_{23}$ & 1.361 & 1.361 & 1.356 & $\mathrm{C}_{8}-\mathrm{C}_{11}-\mathrm{C}_{10}$ & 120.0 & 120.0 & 120.0 & $\mathrm{C}_{3}-\mathrm{C}_{4}-\mathrm{C}_{9}-\mathrm{C}_{10}$ & 0.987 & -0.994 & -0.675 \\
\hline $\mathrm{C}_{21}-\mathrm{F}_{24}$ & 1.349 & 1.348 & 1.345 & $\mathrm{C}_{8}-\mathrm{C}_{11}-\mathrm{H}_{12}$ & 120.3 & 120.3 & 120.3 & $\mathrm{C}_{3}-\mathrm{C}_{4}-\mathrm{C}_{9}-\mathrm{C}_{21}$ & -179.2 & 179.2 & 179.5 \\
\hline & & & & $\mathrm{C}_{10}-\mathrm{C}_{11}-\mathrm{H}_{12}$ & 119.6 & 119.6 & 119.6 & $\mathrm{~N}_{13}-\mathrm{C}_{4}-\mathrm{C}_{9}-\mathrm{C}_{10}$ & -178.6 & 178.6 & 179.1 \\
\hline & & & & $\mathrm{C}_{4}-\mathrm{N}_{13}-\mathrm{C}_{5}$ & 117.8 & 117.8 & 118.4 & $\mathrm{~N}_{13}-\mathrm{C}_{4}-\mathrm{C}_{9}-\mathrm{C}_{21}$ & 1.015 & -1.031 & -0.678 \\
\hline & & & & $\mathrm{C}_{2}-\mathrm{N}_{14}-\mathrm{H}_{15}$ & 116.2 & 116.2 & 117.9 & $\mathrm{C}_{3}-\mathrm{C}_{4}-\mathrm{N}_{13}-\mathrm{C}_{5}$ & -0.026 & 0.029 & -0.054 \\
\hline & & & & $\mathrm{C}_{2}-\mathrm{N}_{14}-\mathrm{H}_{16}$ & 115.0 & 115.0 & 116.3 & $\mathrm{C}_{9}-\mathrm{C}_{4}-\mathrm{N}_{13}-\mathrm{C}_{5}$ & 179.6 & -179.6 & -179.8 \\
\hline & & & & $\mathrm{H}_{15}-\mathrm{N}_{14}-\mathrm{H}_{16}$ & 111.8 & 111.8 & 113.4 & $\mathrm{C}_{1}-\mathrm{C}_{5}-\mathrm{N}_{13}-\mathrm{C}_{4}$ & 1.244 & -1.261 & -0.924 \\
\hline & & & & $\mathrm{C}_{5}-\mathrm{C}_{17}-\mathrm{H}_{18}$ & 110,8 & 111.0 & 111.1 & $\mathrm{C}_{17}-\mathrm{C}_{5}-\mathrm{N}_{13}-\mathrm{C}_{4}$ & -179.3 & 179.3 & 179.2 \\
\hline & & & & $\mathrm{C}_{5}-\mathrm{C}_{17}-\mathrm{H}_{19}$ & 111.0 & 110.8 & 110.6 & $\mathrm{C}_{1}-\mathrm{C}_{5}-\mathrm{C}_{17}-\mathrm{H}_{18}$ & -61.64 & -57.35 & -52.99 \\
\hline
\end{tabular}




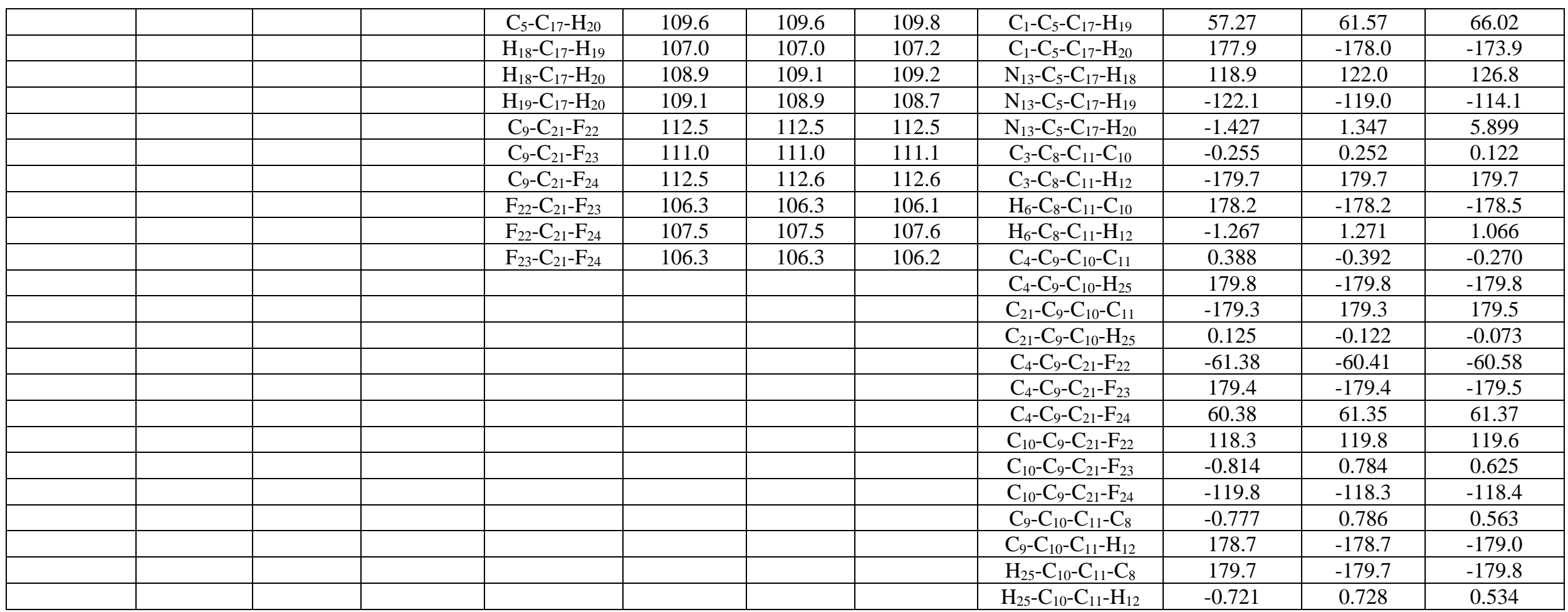

Table 2. Vibrational assignment of 4-amino-2-methyl-8-(trifluoromethyl)quinoline by normal mode analysis based on SQM force field calculations.

\begin{tabular}{|c|c|c|c|c|c|c|c|c|c|}
\hline \multicolumn{7}{|c|}{ Theoretical (B3LYP) } & \multicolumn{2}{|c|}{ Experimental } & \multirow[t]{3}{*}{$\operatorname{PED}(\%)^{\mathrm{c}}$} \\
\hline & & \multicolumn{3}{|c|}{ cc-pVDZ } & cc-pVTZ & cc-pVQZ & & & \\
\hline \multicolumn{2}{|c|}{ Normal Modes } & Freq. $^{\mathrm{a}}$ & $\mathrm{I}_{\mathrm{IR}}^{\mathrm{b}}$ & $\mathrm{I}_{\text {Raman }}{ }^{\mathrm{b}}$ & Freq. $^{\text {a }}$ & Freq. $^{\text {a }}$ & FT-IR & FT-Raman & \\
\hline $\mathrm{A}^{\prime \prime}$ & $v_{1}$ & 54 & 0.020 & 0.476 & 54 & 36 & & & $\tau_{\mathrm{CCCC}}(10)+\tau_{\mathrm{CCCH}}(17)+\tau_{\mathrm{CCCN}}(13)+\tau_{\mathrm{CCHN}}(10)+\tau_{\mathrm{CCCF}}(36)$ \\
\hline $\mathrm{A}^{\prime \prime}$ & $v_{2}$ & 59 & 0.100 & 1.105 & 58 & 57 & & & $\tau_{\mathrm{CCCC}}(20)+\tau_{\mathrm{CCCH}}(19)+\tau_{\mathrm{CCCN}}(18)+\tau_{\mathrm{CCHN}}(13)+\tau_{\mathrm{CCCF}}(20)$ \\
\hline $\mathrm{A}^{\prime \prime}$ & $v_{3}$ & 67 & 0.231 & 0.048 & 66 & 60 & & & $\tau_{\mathrm{CCCH}}(43)+\tau_{\mathrm{CCHN}}(44)$ \\
\hline $\mathrm{A}^{\prime \prime}$ & $v_{4}$ & 129 & 0.295 & 0.557 & 128 & 129 & & & $\tau_{\mathrm{CCCC}}(18)+\tau_{\mathrm{CCCH}}(22)+\tau_{\mathrm{CCCN}}(18)+\tau_{\mathrm{CCHN}}(12)+\tau_{\mathrm{CCCF}}(9)$ \\
\hline $\mathrm{A}^{\prime}$ & $v_{5}$ & 132 & 0.257 & 0.429 & 131 & 132 & & & $\tau_{\mathrm{CCCC}}(18)+\tau_{\mathrm{CCCH}}(22)+\tau_{\mathrm{CCCN}}(19)+\tau_{\mathrm{CCHN}}(12)+\tau_{\mathrm{CCCF}}(10)$ \\
\hline $\mathrm{A}^{\prime \prime}$ & $v_{6}$ & 168 & 1.242 & 0.501 & 167 & 165 & & $148 \mathrm{~m}$ & $\tau_{\mathrm{CCCC}}(29)+\tau_{\mathrm{CCCH}}(17)+\tau_{\mathrm{CCCN}}(23)+\tau_{\mathrm{CCCF}}(15)$ \\
\hline $\mathrm{A}^{\prime \prime}$ & $v_{7}$ & 184 & 0.318 & 0.279 & 183 & 183 & & $199 \mathrm{~m}$ & $\tau_{\mathrm{CCCC}}(31)+\tau_{\mathrm{CCCH}}(12)+\tau_{\mathrm{CCCN}}(30)+\tau_{\mathrm{CCHN}}(10)$ \\
\hline
\end{tabular}




\begin{tabular}{|c|c|c|c|c|c|c|c|c|c|}
\hline $\mathrm{A}^{\prime \prime}$ & $v_{8}$ & 260 & 0.142 & 0.781 & 259 & 262 & & $245 \mathrm{~m}$ & $\delta_{\mathrm{CCC}}(19)+\delta_{\mathrm{CCN}}(11)+\delta_{\mathrm{CCF}}(10)+\tau_{\mathrm{CCNH}}(17)$ \\
\hline $\mathrm{A}^{\prime}$ & $v_{9}$ & 276 & 0.259 & 0.392 & 274 & 276 & & & $\tau_{\mathrm{CCCC}}(24)+\tau_{\mathrm{CCCH}}(22)+\tau_{\mathrm{CCCN}}(23)$ \\
\hline A" & $v_{10}$ & 285 & 0.847 & 1.144 & 284 & 289 & & $283 \mathrm{~m}$ & $\tau_{\mathrm{CCCC}}(10)+\tau_{\mathrm{CCCH}}(11)+\tau_{\mathrm{CCCN}}(14)+\tau_{\mathrm{CCNH}}(25)$ \\
\hline $\mathrm{A}^{\prime}$ & $v_{11}$ & 309 & 1.552 & 0.394 & 307 & 314 & & $310 \mathrm{~s}$ & $\tau_{\mathrm{CCCC}}(10)+\tau_{\mathrm{CCCH}}(10)+\tau_{\mathrm{CCNH}}(39)$ \\
\hline $\mathrm{A}^{\prime}$ & $v_{12}$ & 330 & 4.215 & 0.308 & 328 & 331 & & $334 \mathrm{~m}$ & $\tau_{\mathrm{CCCC}}(12)+\tau_{\mathrm{CCNH}}(41)$ \\
\hline $\mathrm{A}^{\prime \prime}$ & $v_{13}$ & 357 & 0.314 & 0.792 & 355 & 358 & & & $\delta_{\mathrm{CCC}}(13)+\tau_{\mathrm{CCCC}}(19)+\tau_{\mathrm{CCCH}}(13)+\tau_{\mathrm{CCCN}}(13)$ \\
\hline $\mathrm{A}^{\prime}$ & $v_{14}$ & 370 & 7.429 & 0.440 & 368 & 374 & & $372 \mathrm{~m}$ & $\tau_{\mathrm{CCCC}}(19)+\tau_{\mathrm{CCCH}}(14)+\tau_{\mathrm{CCCN}}(14)+\tau_{\mathrm{CCNH}}(33)$ \\
\hline A" & $v_{15}$ & 453 & 0.413 & 3.426 & 451 & 454 & & & $v_{\mathrm{CC}}(13)+\delta_{\mathrm{CCC}}(17)+\delta_{\mathrm{CCH}}(10)+\delta_{\mathrm{CCN}}(17)$ \\
\hline $\mathrm{A}^{\prime}$ & $v_{16}$ & 478 & 7.758 & 0.941 & 475 & 473 & & $465 \mathrm{~s}$ & $\tau_{\mathrm{CCCC}}(24)+\tau_{\mathrm{CCCH}}(18)+\tau_{\mathrm{CCCN}}(16)+\tau_{\mathrm{CCNH}}(12)+\tau_{\mathrm{CCCF}}(12)$ \\
\hline A" & $v_{17}$ & 495 & 0.372 & 1.551 & 492 & 492 & & & $\delta_{\mathrm{CCC}}(14)+\delta_{\mathrm{CCH}}(10)+\delta_{\mathrm{CCN}}(13)+\delta_{\mathrm{CCF}}(10)+\tau_{\mathrm{CCCF}}(16)$ \\
\hline $\mathrm{A}^{\prime}$ & $v_{18}$ & 524 & 37.43 & 1.614 & 521 & 496 & & $500 \mathrm{~m}$ & $\tau_{\mathrm{CCCC}}(17)+\tau_{\mathrm{CCCH}}(11)+\tau_{\mathrm{CCCN}}(10)+\tau_{\mathrm{CCNH}}(27)$ \\
\hline $\mathrm{A}^{\prime}$ & $v_{19}$ & 528 & 1.548 & 0.783 & 525 & 527 & & $511 \mathrm{~m}$ & $\tau_{\mathrm{CCCC}}(22)+\tau_{\mathrm{CCCH}}(21)+\tau_{\mathrm{CCCN}}(21)$ \\
\hline A" & $v_{20}$ & 535 & 11.29 & 1.303 & 532 & 534 & & $546 \mathrm{~m}$ & $\delta_{\mathrm{CCC}}(11)+\tau_{\mathrm{CCCC}}(10)+\tau_{\mathrm{CCCH}}(12)+\tau_{\mathrm{CCCN}}(11)+\tau_{\mathrm{CCNH}}(17)$ \\
\hline $\mathrm{A}^{\prime}$ & $v_{21}$ & 546 & 16.48 & 3.182 & 543 & 545 & $563 \mathrm{~m}$ & $561 \mathrm{~m}$ & $\delta_{\mathrm{CCC}}(11)+\tau_{\mathrm{CCCC}}(14)+\tau_{\mathrm{CCNH}}(22)$ \\
\hline A" & $v_{22}$ & 584 & 7.882 & 0.513 & 581 & 581 & $580 \mathrm{vw}$ & & $\tau_{\mathrm{CCCC}}(24)+\tau_{\mathrm{CCCH}}(17)+\tau_{\mathrm{CCCN}}(11)+\tau_{\mathrm{CCNH}}(17)$ \\
\hline $\mathrm{A}^{\prime}$ & $v_{23}$ & 590 & 5.028 & 1.585 & 587 & 590 & & & $\delta_{\mathrm{CCC}}(15)+\tau_{\mathrm{CCCC}}(15)+\tau_{\mathrm{CCCH}}(10)$ \\
\hline A" & $v_{24}$ & 625 & 5.617 & 0.533 & 622 & 625 & $605 \mathrm{~s}$ & $607 \mathrm{~m}$ & $\tau_{\mathrm{CCCC}}(15)+\tau_{\mathrm{CCCH}}(20)+\tau_{\mathrm{CCCN}}(30)+\tau_{\mathrm{CCNH}}(12)$ \\
\hline $\mathrm{A}^{\prime}$ & $v_{25}$ & 667 & 4.708 & 1.625 & 663 & 669 & & & $\delta_{\mathrm{CCC}}(15)+\delta_{\mathrm{CCH}}(10)+\delta_{\mathrm{CCN}}(10)+\tau_{\mathrm{CCCN}}(10)$ \\
\hline $\mathrm{A}^{\prime \prime}$ & $v_{26}$ & 692 & 0.685 & 0.198 & 689 & 692 & $679 \mathrm{vs}$ & $684 \mathrm{~m}$ & $\tau_{\mathrm{CCCC}}(24)+\tau_{\mathrm{CCCH}}(18)+\tau_{\mathrm{CCCN}}(16)+\tau_{\mathrm{CCNH}}(12)+\tau_{\mathrm{CCCF}}(12)$ \\
\hline $\mathrm{A}^{\prime}$ & $v_{27}$ & 715 & 0.704 & 2.694 & 711 & 716 & $732 \mathrm{w}$ & $735 \mathrm{~m}$ & $v_{\mathrm{CC}}(15)+\delta_{\mathrm{CCC}}(16)+\delta_{\mathrm{CCH}}(10)+\delta_{\mathrm{CCN}}(10)$ \\
\hline A" & $v_{28}$ & 755 & 8.877 & 0.719 & 751 & 754 & $771 \mathrm{vs}$ & $762 \mathrm{~m}$ & $\tau_{\mathrm{CCCC}}(13)+\tau_{\mathrm{CCCH}}(45)+\tau_{\mathrm{CCCN}}(20)$ \\
\hline $\mathrm{A}^{\prime}$ & $v_{29}$ & 818 & 3.869 & 0.901 & 814 & 820 & & & $\tau_{\mathrm{CCCC}}(22)+\tau_{\mathrm{CCCH}}(24)+\tau_{\mathrm{CCCN}}(13)+\tau_{\mathrm{CCNH}}(10)$ \\
\hline $\mathrm{A}^{\prime \prime}$ & $v_{30}$ & 821 & 4.431 & 1.664 & 816 & 825 & $825 \mathrm{vs}$ & & $\tau_{\mathrm{CCCC}}(16)+\tau_{\mathrm{CCCH}}(22)+\tau_{\mathrm{CCCN}}(12)+\tau_{\mathrm{CCNH}}(11)$ \\
\hline $\mathrm{A}^{\prime}$ & $v_{31}$ & 829 & 3.605 & 0.861 & 825 & 839 & $842 \mathrm{~s}$ & $836 \mathrm{vw}$ & $\tau_{\mathrm{CCCC}}(21)+\tau_{\mathrm{CCCH}}(25)+\tau_{\mathrm{CCCN}}(16)+\tau_{\mathrm{CCNH}}(24)$ \\
\hline $\mathrm{A}^{\prime \prime}$ & $v_{32}$ & 861 & 3.162 & 0.574 & 856 & 862 & $872 \mathrm{~m}$ & & $v_{\mathrm{CC}}(14)+\delta_{\mathrm{CCC}}(22)+\delta_{\mathrm{CCH}}(20)+\delta_{\mathrm{CCN}}(10)$ \\
\hline $\mathrm{A}^{\prime}$ & $v_{33}$ & 915 & 0.850 & 0.344 & 910 & 914 & $930 \mathrm{w}$ & & $\tau_{\mathrm{CCCC}}(14)+\tau_{\mathrm{CCCH}}(52)+\tau_{\mathrm{CCHN}}(16)$ \\
\hline $\mathrm{A}^{\prime \prime}$ & $v_{34}$ & 962 & 0.642 & 0.310 & 957 & 959 & & & $\tau_{\mathrm{CCCC}}(15)+\tau_{\mathrm{CCCH}}(56)+\tau_{\mathrm{CCHH}}(15)$ \\
\hline $\mathrm{A}^{\prime}$ & $v_{35}$ & 967 & 3.711 & 0.394 & 962 & 971 & & & $v_{\mathrm{CC}}(9)+\delta_{\mathrm{CCC}}(12)+\delta_{\mathrm{CCH}}(22)+\delta_{\mathrm{CCN}}(10)+\tau_{\mathrm{CCCH}}(13)+\tau_{\mathrm{CCHN}}(10)$ \\
\hline $\mathrm{A}^{\prime}$ & $v_{36}$ & 981 & 7.069 & 0.103 & 976 & 984 & $993 \mathrm{~s}$ & $993 \mathrm{~m}$ & $v_{\mathrm{CC}}(13)+\delta_{\mathrm{CCC}}(13)+\delta_{\mathrm{CCH}}(20)+\delta_{\mathrm{CCN}}(10)+\tau_{\mathrm{CCCH}}(10)$ \\
\hline $\mathrm{A}^{\prime \prime}$ & $v_{37}$ & 1012 & 0.751 & 0.012 & 1007 & 1026 & & & $\delta_{\mathrm{CCH}}(20)+\tau_{\mathrm{CCCH}}(27)+\tau_{\mathrm{CCCN}}(18)+\tau_{\mathrm{CCHN}}(10)$ \\
\hline $\mathrm{A}^{\prime}$ & $v_{38}$ & 1057 & 6.566 & 2.253 & 1052 & 1051 & & & $v_{\mathrm{CC}}(13)+\delta_{\mathrm{CCC}}(12)+\delta_{\mathrm{CCH}}(25)+\delta_{\mathrm{CN}}(11)$ \\
\hline A" & $v_{39}$ & 1065 & 12.98 & 1.076 & 1060 & 1062 & & & $v_{\mathrm{CC}}(18)+\delta_{\mathrm{CCC}}(11)+\delta_{\mathrm{CCH}}(24)$ \\
\hline $\mathrm{A}^{\prime}$ & $v_{40}$ & 1097 & 18.23 & 1.021 & 1091 & 1085 & $1076 \mathrm{vs}$ & $1077 \mathrm{~m}$ & $v_{\mathrm{CC}}(15)+\delta_{\mathrm{CCC}}(11)+\delta_{\mathrm{CCH}}(18)+\delta_{\mathrm{CN}}(14)$ \\
\hline
\end{tabular}




\begin{tabular}{|c|c|c|c|c|c|c|c|c|c|}
\hline $\mathrm{A}^{\prime}$ & $v_{41}$ & 1130 & 30.2 & 0.562 & 1124 & 1106 & & & $v_{\mathrm{CC}}(13)+\delta_{\mathrm{CCC}}(11)+\delta_{\mathrm{CCH}}(19)+\tau_{\mathrm{CCCF}}(14)$ \\
\hline $\mathrm{A}^{\prime}$ & $v_{42}$ & 1149 & 76.91 & 0.675 & 1143 & 1112 & 1117 vs & & $v_{\mathrm{CF}}(22)+\delta_{\mathrm{CCF}}(10)+\tau_{\mathrm{CCCC}}(12)+\tau_{\mathrm{CCCF}}(24)$ \\
\hline $\mathrm{A}^{\prime}$ & $v_{43}$ & 1155 & 3.657 & 1.666 & 1149 & 1161 & $1143 \mathrm{~s}$ & $1144 \mathrm{~m}$ & $v_{\mathrm{CC}}(11)+\delta_{\mathrm{CCC}}(10)+\delta_{\mathrm{CCH}}(45)$ \\
\hline $\mathrm{A}^{\prime}$ & $v_{44}$ & 1163 & 11.66 & 0.434 & 1157 & 1170 & $1189 \mathrm{~m}$ & $1182 \mathrm{~m}$ & $v_{\mathrm{CC}}(13)+\delta_{\mathrm{CCC}}(10)+\delta_{\mathrm{CCH}}(37)$ \\
\hline $\mathrm{A}^{\prime}$ & $v_{45}$ & 1202 & 4.782 & 0.757 & 1196 & 1209 & $1228 \mathrm{~m} . \mathrm{sh}$ & $1228 \mathrm{~m}$ & $v_{\mathrm{CC}}(15)+\delta_{\mathrm{CCC}}(20)+\delta_{\mathrm{CCH}}(28)+\delta_{\mathrm{CCN}}(9)$ \\
\hline $\mathrm{A}^{\prime}$ & $v_{46}$ & 1256 & 0.527 & 0.857 & 1249 & 1252 & & $1259 \mathrm{~m}$ & $v_{\mathrm{CC}}(23)+\delta_{\mathrm{CCC}}(12)+\delta_{\mathrm{CCH}}(26)$ \\
\hline $\mathrm{A}^{\prime \prime}$ & $v_{47}$ & 1281 & 100 & 8.203 & 1274 & 1266 & $1308 \mathrm{vs}$ & & $v_{\mathrm{CC}}(19)+v_{\mathrm{CF}}(19)+\delta_{\mathrm{CCC}}(14)+\delta_{\mathrm{CCH}}(26)$ \\
\hline $\mathrm{A}^{\prime}$ & $v_{48}$ & 1328 & 8.533 & 3.011 & 1321 & 1320 & & $1309 \mathrm{~s}$ & $v_{\mathrm{CC}}(21)+\delta_{\mathrm{CCC}}(10)+\delta_{\mathrm{CCH}}(23)$ \\
\hline $\mathrm{A}^{\prime}$ & $v_{49}$ & 1338 & 0.613 & 7.879 & 1331 & 1333 & $1332 \mathrm{vw}$ & & $v_{\mathrm{CC}}(17)+\delta_{\mathrm{CCH}}(32)+\delta_{\mathrm{HCH}}(24)$ \\
\hline $\mathrm{A}^{\prime}$ & $v_{50}$ & 1360 & 6.484 & 54.59 & 1353 & 1357 & $1353 \mathrm{~m} . \mathrm{sh}$ & 1352 vs & $v_{\mathrm{CC}}(35)+\delta_{\mathrm{CCC}}(14)+\delta_{\mathrm{CCH}}(20)$ \\
\hline $\mathrm{A}^{\prime}$ & $v_{51}$ & 1383 & 6.340 & 2.339 & 1376 & 1377 & & $1375 \mathrm{w}$ & $v_{\mathrm{CC}}(14)+\delta_{\mathrm{CCH}}(14)+\delta_{\mathrm{CN}}(16)+\delta_{\mathrm{HCH}}(19)+\tau_{\mathrm{CCCH}}(10)+\tau_{\mathrm{CCHN}}(10)$ \\
\hline $\mathrm{A}^{\prime}$ & $v_{52}$ & 1401 & 6.917 & 22.29 & 1394 & 1423 & $1421 \mathrm{vw}$ & $1421 \mathrm{~m}$ & $v_{\mathrm{CC}}(11)+\delta_{\mathrm{CCH}}(14)+\delta_{\mathrm{HCH}}(23)+\tau_{\mathrm{CCCH}}(13)+\tau_{\mathrm{CCHN}}(13)$ \\
\hline $\mathrm{A}^{\prime}$ & $v_{53}$ & 1411 & 1.967 & 5.709 & 1404 & 1428 & & $1434 \mathrm{~m}$ & $\delta_{\mathrm{CCH}}(9)+\delta_{\mathrm{HCH}}(34)+\tau_{\mathrm{CCCH}}(24)+\tau_{\mathrm{CCHN}}(24)$ \\
\hline $\mathrm{A}^{\prime}$ & $v_{54}$ & 1421 & 2.239 & 17.66 & 1414 & 1441 & $1442 \mathrm{~m}$ & $1441 \mathrm{~m}$ & $v_{\mathrm{CC}}(22)+\delta_{\mathrm{CCC}}(19)+\delta_{\mathrm{CCH}}(27)$ \\
\hline A" & $v_{55}$ & 1462 & 2.961 & 1.354 & 1454 & 1462 & & & $v_{\mathrm{CC}}(22)+\delta_{\mathrm{CCC}}(13)+\delta_{\mathrm{CCH}}(32)$ \\
\hline $\mathrm{A}^{\prime}$ & $v_{56}$ & 1510 & 17.10 & 0.910 & 1502 & 1501 & $1517 \mathrm{~s}$ & $1525 \mathrm{~m}$ & $v_{\mathrm{CC}}(26)+\delta_{\mathrm{CCC}}(12)+\delta_{\mathrm{CCH}}(30)$ \\
\hline $\mathrm{A}^{\prime}$ & $v_{57}$ & 1571 & 24.87 & 13.62 & 1563 & 1557 & 1569 vs & $1572 \mathrm{~s}$ & $v_{\mathrm{CC}}(34)+\delta_{\mathrm{CCC}}(18)+\delta_{\mathrm{CCH}}(19)+\delta_{\mathrm{CCN}}(11)$ \\
\hline $\mathrm{A}^{\prime}$ & $v_{58}$ & 1584 & 14.86 & 2.581 & 1576 & 1585 & 1592 vs & $1594 \mathrm{~m}$ & $v_{\mathrm{CC}}(16)+\delta_{\mathrm{CCC}}(10)+\delta_{\mathrm{CCH}}(13)+\delta_{\mathrm{CNH}}(12)+\delta_{\mathrm{HNH}}(14)+\tau_{\mathrm{CCNH}}(15)$ \\
\hline $\mathrm{A}^{\prime}$ & $v_{59}$ & 1609 & 36.23 & 4.025 & 1601 & 1603 & $1619 \mathrm{~s}$ & $1621 \mathrm{~m}$ & $v_{\mathrm{CC}}(21)+\delta_{\mathrm{CCC}}(13)+\delta_{\mathrm{CCH}}(22)+\tau_{\mathrm{CCNH}}(11)$ \\
\hline $\mathrm{A}^{\prime}$ & $v_{60}$ & 1619 & 46.79 & 12.29 & 1611 & 1615 & $1638 \mathrm{~s}$ & $1637 \mathrm{~m}$ & $v_{\mathrm{CC}}(27)+\delta_{\mathrm{CCC}}(17)+\delta_{\mathrm{CCH}}(16)$ \\
\hline $\mathrm{A}^{\prime}$ & $v_{61}$ & 2939 & 5.973 & 100 & 2924 & 2933 & $2924 \mathrm{~m}$ & $2925 \mathrm{~m}$ & $v_{\mathrm{CH}}(88)$ \\
\hline $\mathrm{A}^{\prime}$ & $v_{62}$ & 2996 & 4.207 & 43.04 & 2980 & 2979 & $2956 \mathrm{vw}$ & $2964 \mathrm{~m}$ & $v_{\mathrm{CH}}(76)$ \\
\hline $\mathrm{A}^{\prime}$ & $v_{63}$ & 3057 & 1.382 & 22.50 & 3041 & 3039 & & & $v_{\mathrm{CH}}(77)$ \\
\hline A" & $v_{64}$ & 3072 & 4.883 & 46.55 & 3056 & 3062 & & & $v_{\mathrm{CH}}(71)$ \\
\hline $\mathrm{A}^{\prime}$ & $v_{65}$ & 3084 & 1.362 & 17.98 & 3068 & 3072 & & $3072 \mathrm{~m}$ & $v_{\mathrm{CH}}(78)+\delta_{\mathrm{CCC}}(10)$ \\
\hline $\mathrm{A}^{\prime}$ & $v_{66}$ & 3104 & 4.864 & 58.35 & 3088 & 3091 & & $3099 \mathrm{vw}$ & $v_{\mathrm{CH}}(81)$ \\
\hline $\mathrm{A}^{\prime}$ & $v_{67}$ & 3128 & 0.717 & 61.78 & 3112 & 3114 & $3252 \mathrm{~m}$ & & $v_{\mathrm{CH}}(79)$ \\
\hline $\mathrm{A}^{\prime}$ & $v_{68}$ & 3443 & 6.661 & 71.51 & 3426 & 3472 & $3383 \mathrm{~s}$ & $3388 \mathrm{~m}$ & $v_{\mathrm{NH}}(87)$ \\
\hline $\mathrm{A}^{\prime}$ & $v_{69}$ & 3541 & 4.892 & 21.71 & 3524 & 3569 & $3494 \mathrm{~s}$ & & $v_{\mathrm{NH}}(84)$ \\
\hline
\end{tabular}

vs: very strong. ms: medium strong. s: strong. w: weak. vw: very weak.v: stretching. t: torsion. $\gamma$ : out of plane stretching. $\delta$ : in plane bending

a Obtained from the wavenumbers calculated at 0.970 for cc-pVDZ. 0.965 for cc-pVTZ. and 0.969 for cc-pVQZ basis sets [36].

${ }^{6}$ Relative absorbtion intensities and Relative Raman activities normalized with highest peak absorption equal to 100.

${ }^{c}$ Total energy distribution calculated B3LYP cc-pVDZ level of theory. Only contributions $\geq 10 \%$ are listed. 


\section{HOMO-LUMO analysis}

HOMO (Highest Occupied Molecular Orbital) and LUMO (Lowest Unoccupied Molecular Orbital) are known as Molecular orbitals. They were very important electronic parameters for chemist and physicist. The LUMO are known as the inner-most orbital containing free places to accept electrons. It defines the ability of accepting electron. HOMO can be considered the outermost orbital containing electrons. It characterizes the ability of giving electron. HOMO and LUMO energies of chemical compounds are important quantum mechanical parameters. They are very useful to explain its reactivity. The plots of HOMO, HOMO-1, and LUMO, LUMO+1, molecular orbitals for the AMTQ were given in Figure 4.

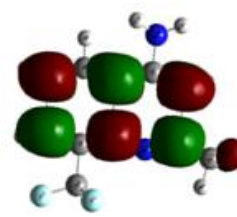
$E_{\text {LUMO }+1}=-0.149 \mathrm{eV}$

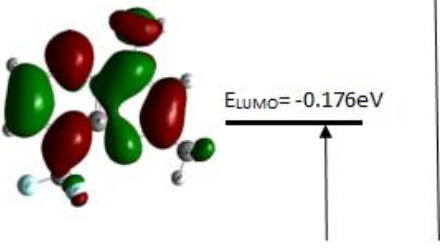

$\Delta \mathrm{E}=0.122 \mathrm{eV} \quad \Delta \mathrm{E}=0.175 \mathrm{eV}$
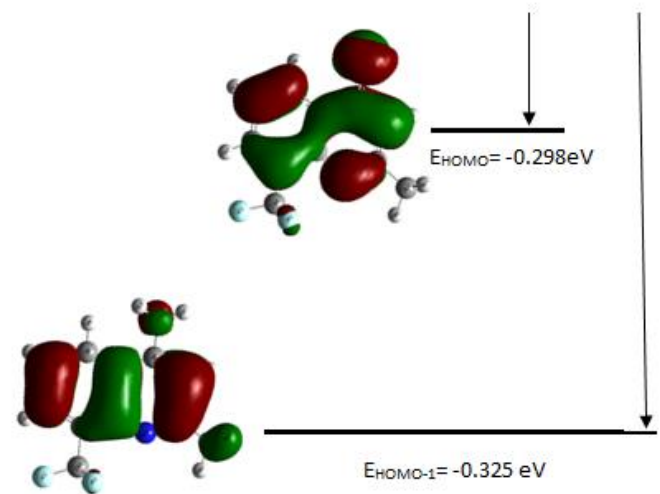

Figure 4. HOMO and LUMO plot of of 4-amino-2methyl-8-(trifluoromethyl)quinolone molecule ( B3LYP / cc-pVDZ)

\section{Nuclear Magnetic Resonance Spectra}

Significant progress has been achieved in chemical shifts calculations which aids in spectral assignment and structural elucidation. Meanwhile, the Gauge Invariant Atomic Orbitals (GIAO) approach [37,38] that uses density functional theory, DFT-B3LYP and DFTB3PW91 calculations have afforded excellent agreement with experimental NMR spectral analysis [39-41]. Therefore, we have used the optimized geometry of AMTQ structure from B3LYP using cc-pVDZ, cc-pVTZ and cc-pVQZ basis sets in DMSO solution for further optimization, while employing Tomasi's Polarized Continuum Model (PCM) [19]. The ${ }^{13} \mathrm{C}$ NMR nuclear shielding constants were predicted using GIAO approximation with the above method. The calculated ${ }^{1} \mathrm{H}$ and ${ }^{13} \mathrm{C}$ absolute isotropic shielding (si, ppm) parameters were converted into ${ }^{1} \mathrm{H}$ and ${ }^{13} \mathrm{C}$ chemical shifts $\left(\delta_{\text {calc }}\right.$, ppm.) using $\left(\delta_{\text {calc }}=\delta_{\mathrm{TMS}}-\delta_{\mathrm{i}}\right)$. The experimental peaks along with the simulated ${ }^{1} \mathrm{H}$ (a) and ${ }^{13} \mathrm{C}$ (b) nuclear magnetic resonance (NMR) spectra of 4-amino-2methyl-8-(trifluoromethyl)quinoline molecule shown in Figure 5.

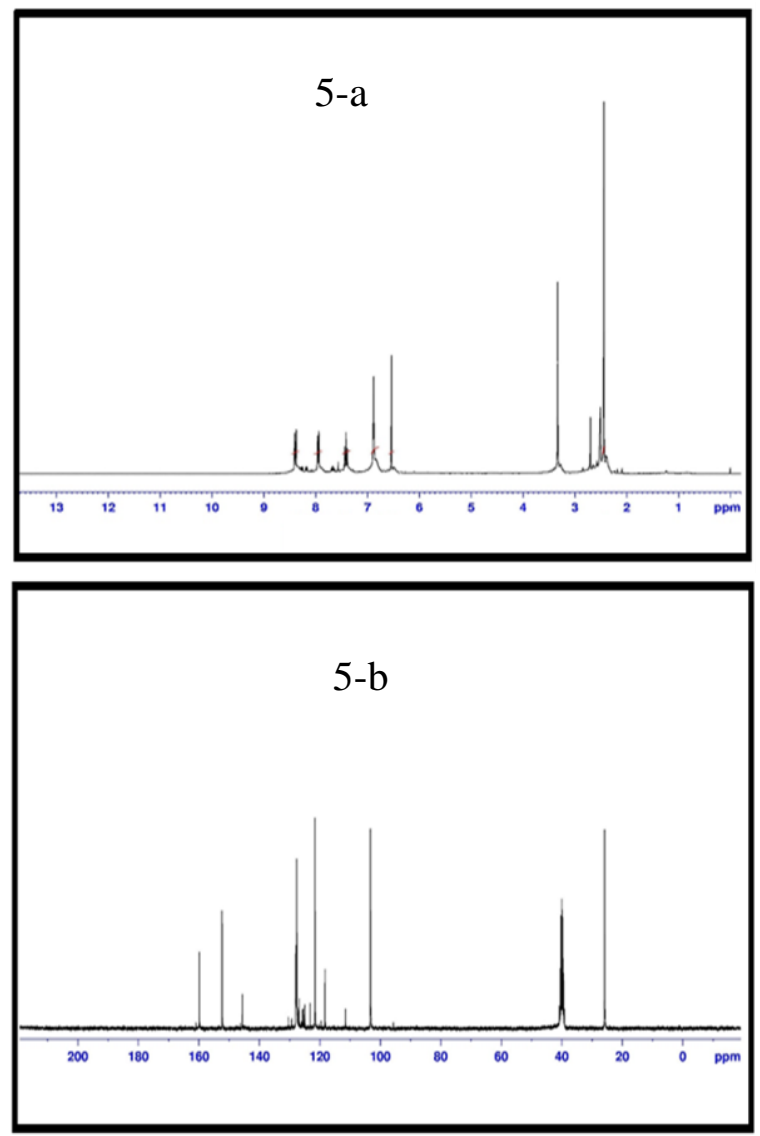

Figure 5. ${ }^{1} \mathrm{H}$ (a) and ${ }^{13} \mathrm{C}$ (b) NMR spectra of 4-amino2-methyl-8-(trifluoromethyl)quinoline

${ }^{1} \mathrm{H}$ and ${ }^{13} \mathrm{C}$ experimental and theoretical NMR chemical shifts are listed in Table 3 with AMTQ sample dissolved in DMSO-d6. According to the ${ }^{13} \mathrm{C}$ NMR spectrum, the signal observed at $160.9 \mathrm{ppm}$ are undoubtedly assigned to the $\mathrm{C}_{5}\left(\mathrm{C}_{5}-\mathrm{C}_{17}-\mathrm{H}_{3}\right)$ which agrees with $\mathrm{B} 3 \mathrm{LYP} / \mathrm{cc}$ pVDZ, cc-pVTZ and cc-pVQZ predicted values at 161.9 ppm, 169.1 ppm and 172.4 ppm, respectively. Similarly, the signals observed at $159.8 \mathrm{ppm}$ have been assigned to $\mathrm{C}_{2}$ and $\mathrm{C}_{2}$ (coordinated to the amino group) in agreement with estimated values at $160.6 \mathrm{ppm}$ using B3LYP/ cc-pVQZ. ${ }^{1} \mathrm{H}$ NMR spectra interpreted significantly in an attempt to measure the possible different effects appearing on the chemical shift values of protons. Observed proton chemical shift values are in the region 
8.406-2.441 ppm. In the proton NMR spectra, the signals are observed at $6.883\left(\mathrm{H}_{7}\right), 7.940\left(\mathrm{H}_{12}\right), 8.379\left(\mathrm{H}_{6}\right)$ and $8.406\left(\mathrm{H}_{25}\right) \mathrm{ppm}$. Hydrogen atoms attached to quinoline ring. The methyl hydrogen atoms are $\mathrm{H}_{18}, \mathrm{H}_{19}$ and $\mathrm{H}_{20}$ and exhibit shift at 2.515, 2.509 and $2.441 \mathrm{ppm}$, respectively. The chemical shifts of the $\mathrm{H}_{15}$ and $\mathrm{H}_{16}$, which is attached to $\mathrm{N}_{14}$, appeared at $3.400 \mathrm{ppm}$ and $3.332 \mathrm{ppm}$ by experimental data. We note that the experimental ${ }^{1} \mathrm{H}$ NMR data agrees with them of the theoretical predicted.

Table 3. Theoretical and experimental ${ }^{1} \mathrm{H}$ and ${ }^{13} \mathrm{C}$ spectra of the title molecule (with respect to TMS, all values in ppm)

\begin{tabular}{|l|l|l|l|l|l|l|l|l|l|}
\hline & \multicolumn{3}{|c|}{ Theoretical (B3LYP) } & Exp. & & \multicolumn{3}{c|}{ Theoretical (B3LYP) } & Exp. \\
\hline & cc-pVDZ & cc-pVTZ & cc-pVQZ & & & cc-pVDZ & cc-pVTZ & cc-pVQZ & \\
\hline $\mathrm{H}_{25}$ & 8.028 & 8.410 & 8.492 & 8.406 & $\mathrm{C}_{5}$ & 161.9 & 169.1 & 172.4 & 160.9 \\
\hline $\mathrm{H}_{6}$ & 7.849 & 8.206 & 8.263 & 8.379 & $\mathrm{C}_{2}$ & 149.4 & 158.2 & 160.6 & 159.8 \\
\hline $\mathrm{H}_{12}$ & 7.430 & 7.723 & 7.770 & 7.940 & $\mathrm{C}_{4}$ & 148.3 & 155.4 & 157.9 & 152.2 \\
\hline $\mathrm{H}_{7}$ & 6.474 & 6.831 & 6.883 & 6.883 & $\mathrm{C}_{9}$ & 134.4 & 139.1 & 142.0 & 145.6 \\
\hline $\mathrm{H}_{15}$ & 3.954 & 4.429 & 4.563 & 3.400 & $\mathrm{C}_{21}$ & 131.4 & 139.3 & 140.1 & 128.0 \\
\hline $\mathrm{H}_{16}$ & 3.385 & 3.896 & 4.064 & 3.332 & $\mathrm{C}_{10}$ & 129.1 & 135.6 & 137.7 & 127.9 \\
\hline $\mathrm{H}_{18}$ & 2.825 & 2.940 & 2.949 & 2.515 & $\mathrm{C}_{8}$ & 124.5 & 130.3 & 132.5 & 127.8 \\
\hline $\mathrm{H}_{19}$ & 2.817 & 2.946 & 2.957 & 2.509 & $\mathrm{C}_{11}$ & 123.4 & 128.8 & 130.8 & 127.6 \\
\hline $\mathrm{H}_{20}$ & 2.651 & 2.925 & 2.980 & 2.441 & $\mathrm{C}_{3}$ & 121.5 & 125.7 & 128.1 & 121.6 \\
\hline & & & & & $\mathrm{C}_{1}$ & 105.4 & 109.3 & 111.0 & 103.2 \\
\hline & & & & $\mathrm{C}_{17}$ & 29.05 & 29.81 & 29.55 & 25.82 \\
\hline
\end{tabular}

\section{Molecular Electrostatic Potential Maps}

The molecular electrostatic potential (MEP) maps of ground state of AMTQ are shown in Fig. 6. In the organic molecules, the MEP is generally used as a reactivity map displaying. These regions are the most probable regions for the electrophilic attack. The MEP contour map provides a simple way to predict how different geometries could interact. The calculated 3D MEP contour map (red is negative, blue is positive) shows the negative regions. In the AMTQ molecule is mainly over the Nitrogen atoms. The electrostatic potential maps have been used for predicting sites.

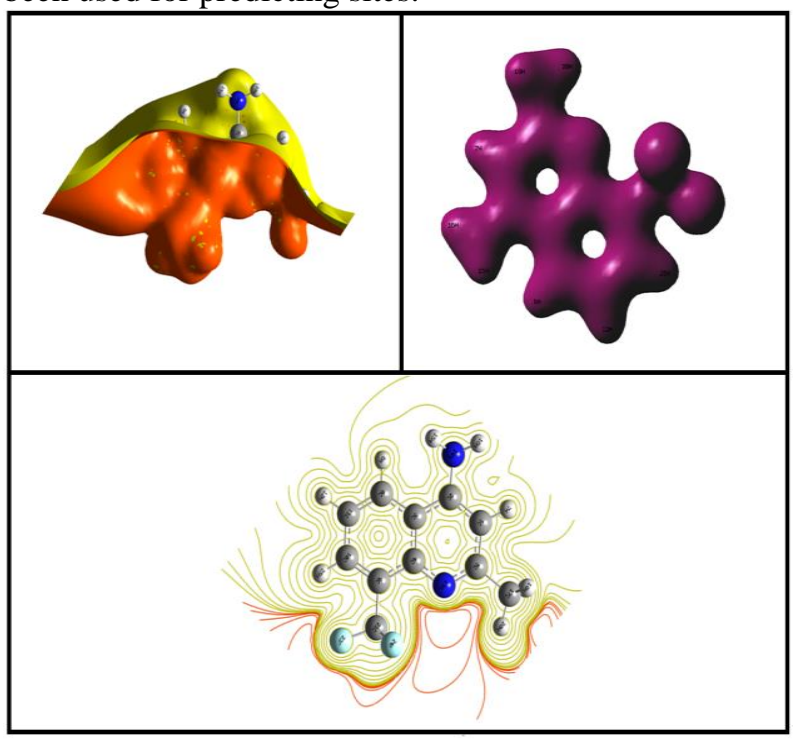

Figure 6. Molecular ESP mapped on the isodensity surface for 4-amino-2-methyl-8-

(trifluoromethyl)quinolone molecule

\section{Conclusion}

This work deal with the vibrational, Nuclear Magnetic Resonance spectra, molecular structure and some electronic properties of AMTQ molecule by theoretical methods and experimental setup. The results of theoretical and experimental studies are combining with together. The most stable conformer of the AMTQ is predicted by DFT calculation. The ground state conformers of AMTQ are further studied using the Density Functional Theory at B3LYP with cc-pVQZ, cc-pVTZ and cc-pVDZ basis sets. The spectroscopic, structural and electronic properties of the ground state have been calculated by Gaussian09. The experimental work has focused on the assignment of the vibrational spectra from 400 to $4000 \mathrm{~cm}^{-1}$. Computed results of the AMTQ are found to be in better agreement with the experimental findings.

\section{References}

1. Quinoline, Wikipedia, https://en.wikipedia.org/wiki/Quinoline, 2017, (accessed 20.05.2017)

2. Wang, L.Y., Chen, Q.W., Zhai, G.H., Wen, Z.Y., Zhang, Z.X. Theoretical study on the structures and absorption properties of styryl dyes with quinoline nucleus, Dyes and Pigmentes, 2007, 72, 357-362.

3. Dahule, H.K., Thejokalyani, N., Dhoble, S.J., Novel Br-DPQ blue light-emitting phosphors for OLED, Luminescence, 2014, 4, 405410 .

4. Ciobotaru, I.C., Polosan, S., Ciobotaru, C.C., Dual emitter IrQ(ppy) 2 for OLED applications: Synthesis and spectroscopic analysis, Journal of Luminescence, 2014, 145, 259-262.

5. Camargo, H, Paolini, T.B., Niyama, E, Brito, H.F., Cremona, M, New rare-earth quinolinate complexes for organic light-emitting devices, Thin Solid Films, 2013, 528, 36-41. 
6. Dereli, O, Erdogdu, Y, Gulluoglu, M.T., Ozmen, A, Sundaraganesan, N, Vibrational spectral and quantum chemical investigations of tert-butyl-hydroquinone, Journal of Molecular Structure, 2012, 1012, 168-176.

7. Dereli, O, Sudha, S, Sundaraganesan, N, Molecular structure and vibrational spectra of 4-phenylsemicarbazide by density functional method, Journal of Molecular Structure, 2011, 994, 379-386.

8. Yurdakul, S, Badoglu, S, An FT-IR and DFT study of the free and solvated 4-(imidazol-1-yl)phenol, Spectrochimica Acta Part A: Molecular and Biomolecular Spectroscopy, 2015, 150, 614-622.

9. Yurdakul, Ş, Badoglu, S, Ozkurt, L, An experimental and theoretical investigation of free Oxazole in conjunction with the DFT analysis of Oxazole $\left(\mathrm{H}_{2} \mathrm{O}\right)_{n}$ complexes, Spectrochimica Acta Part A: Molecular and Biomolecular Spectroscopy, 2016, 162, 4860.

10. Erdogdu, Y, Investigations of FT-IR, FT-Raman, FT-NMR spectra and quantum chemical computations of Esculetin molecule, Spectrochimica Acta Part A: Molecular and Biomolecular Spectroscopy, 2013, 106, 25-33.

11. Erdogdu, Y, Unsalan, O, Gulluoglu, M.T., FT-Raman, FT-IR spectral and DFT studies on 6, 8-dichloroflavone and 6, 8dibromoflavone, Journal of Raman Spectroscopy, 2010, 41, 820828.

12. Subashchandrabose, S, Saleem, H, Erdogdu, Y, Dereli, Ö, Thanikachalam, V, Jayabharathi, J, Structural, vibrational and hyperpolarizability calculation of (E)-2-(2hydroxybenzylideneamino)-3-methylbutanoic acid, Spectrochimica Acta Part A: Molecular and Biomolecular Spectroscopy, 2012, 86, 231-241.

13. Sajan, D, Erdogdu, Y, Kuruvilla, T, Hubert Joe, I, Vibrationa spectra and first-order molecular hyperpolarizabilities of $\mathrm{p}$ hydroxybenzaldehyde dimer, Journal of Molecular Structure, $2010,983,12-21$

14. Erdogdu, Y, Unsalan, O, Sajan, D, Gulluoglu, M.T., Structural conformations and vibrational spectral study of chloroflavone with density functional theoretical simulations, Spectrochimica Acta Part A: Molecular and Biomolecular Spectroscopy, 2010, 76, 130136.

15. Erdoğdu, Y, Güllüoğlu, M.T., Yurdakul, S, Molecular structure and vibrational spectra of 1,3-bis(4-piperidyl)propane by quantum chemical calculations, Journal of Molecular Structure, 2008, 889, $361-370$

16. Frosch, T, Schmitt, M, Popp, J, Raman spectroscopic investigation of the antimalarial agent mefloquine, Analytical and Bioanalytical Chemistry, 2007, 387, 1749-1757.

17. Frosch, T, Popp, J, Structural analysis of the antimalarial drug halofantrine by means of Raman spectrocopy and density functional theory calculations, Journal of Biomedical Optics, 2010, 15 (4), 041516. doi:10.1117/1.3432656

18. Frosch, T, Schmitt, M, Schenzel, K, Faber, J.H., Bringmann, G, Kiefer, W, Popp, J, In vivo localization and identification of the antiplasmodial alkaloid dioncophylline $\mathrm{A}$ in the tropical liana Triphyophyllum peltatum by a combination of fluorescence, near infrared Fourier transform Raman microscopy, and density functional theory calculations, Biopolymers, 2006, 82, 295-300.

19. Frosch, T, Küstner, B, Schlücker, S, Szeghalmi, A, Schmitt, M, Kiefer, W, Popp, J, Invitro polarization-resolved resonance Raman studies of the interaction of hematin with the antimalarial drug chloroquine, Journal of Raman Spectroscopy, 2004, 35, 819-821.
20. Ulahannan, R.T., Panicker, C.Y., Varghese, H.T., Van Alsenoy, C, Musiol, R, Jampilek, J, Anto, P.L, Spectroscopic (FT-IR, FTRaman) investigations and quantum chemical calculations of 4hydroxy-2-oxo-1,2-dihydroquinoline-7-carboxylic acid, Spectrochimica Acta Part A: Molecular and Biomolecular Spectroscopy, 2014, 121, 404-414.

21. Frosch, T, Popp, J, Relationship between molecular structure and Raman spectra of quinolones, Journal of Molecular Structure, 2009, 924-926, 301-308.

22. Cînta-Pînzaru, S, Peica, N, Küstner, B, Schlücker, S, Schmitt, M, Frosch, T, Faber, J.H., Bringmann, G, Popp, J, FT-Raman and NIR-SERS characterization of the antimalarial drugs chloroquine and mefloquine and their interaction with hematin, Journal of Raman Spectroscopy, 2006, 37, 326-334.

23. Frosch, T, Schmitt, M, Bringmann, G, Kiefer, W, Popp, J, Structural Analysis of the Anti-Malaria Active Agent Chloroquine under Physiological Conditions, Journal of Physical Chemistry B, 2007, 111, 1815-1822.

24. Frosch, T, Schmitt, M, Popp, J, In situ UV Resonance Raman Micro-spectroscopic Localization of the Antimalarial Quinine in Cinchona Bark, Journal of Physical Chemistry B, 2007, 111, 41714177 .

25. Fazal, E, Jasinski, J, Anderson, B, Kaur, M, Nagarajan, S, Sudha, B, Synthesis, Crystal and Molecular Structure Studies and DFT Calculations of Phenyl Quinoline-2-Carboxylate and 2Methoxyphenyl Quinoline-2-Carboxylate; Two New Quinoline-2 Carboxylic Derivatives, Crystals, 2015, 5, 100- 115.

26. Diwaker, C.S., Chidan, Kumar, A, Chandraju, Kumar, S, Spectroscopic (FT-IR, ${ }^{1} \mathrm{H},{ }^{13} \mathrm{C}$ NMR and UV-vis) characterization and DFT studies of novel 8-((4-(methylthio)-2,5-diphenylfuran, Spectrochimica Acta Part A: Molecular and Biomolecular Spectroscopy, 2015, 150, 602-613.

27. Ulahannan, R.T., Panicker, C.Y., Varghese, H.T., Musiol, R., Jampilek, J., Alsenoy, C.V., War, J.A., Manojkumar T.K., Vibrational spectroscopic studies and molecular docking study of 2-[(E)-2-phenylethenyl]quinoline-5-carboxylic acid, Spectrochimica Acta Part A: Molecular and Biomolecular Spectroscopy, 2015, 150, 190-199.

28. Kulkarni, A, King, C, Butcher, R.J., Fortunak, M.D., 4,7-Dichloroquinolone, Acta Crystallographica, 2012, E68, 1498.

29. Pereira, G.R., et al., 7-Chloroquinolinotriazoles: Synthesis by the azide-alkyne cycloaddition click chemistry, antimalarial activity, cytotoxicity and SAR studies, European Journal of Medicinal Chemistry, 2014, 73, 295-309.

30. Frisch, M.J., Trucks, G.W., Schlegel, H.B., Scuseria, G.E., Robb, M.A., Cheeseman, J.R., et al., Gaussian 09, revision A.2. Wallingford CT: Gaussian, Inc., 2009.

31. Pulay, P., Baker, J., Wolinski, K, Green Acres Road Suite A Fayettevile, Arkansas, 72703, USA, 2013.

32. Thanikachalam, V, Periyanayagasamy, V, Jayabharathi, J, Manikandan, G, Saleem, H, Subashchandrabose, S, Erdogdu, Y, FT-Raman, FT-IR spectral and DFT studies on (E)-1-4nitrobenzylidenethiocarbonohydrazide, Spectrochimica Acta Part A: Molecular and Biomolecular Spectroscopy, 2012, 87, 86-95.

33. Roeges, N.P.G., A Guide to the Complete Interpretation of Infrared Spectra of Organic Structures, Wiley, New York, 1994.

34. Güllüoğlu, M.T., Erdogdu, Y, Yurdakul, Ş, Molecular structure and vibrational spectra of piperidine and 4-methylpiperidine by 
Celal Bayar University Journal of Science

Volume 13, Issue 4, p 851-861

density functional theory and ab initio Hartree-Fock calculations, Journal of Molecular Structure, 2007, 834, 540-547.

35. Erdogdu, Y, Güllüoğlu, M.T., Analysis of vibrational spectra of 2 and 3-methylpiperidine based on density functional theory calculations, Spectrochimica Acta Part A: Molecular and Biomolecular Spectroscopy, 2009, 74, 162-167.

36. CCCBDB listing of precalculated vibrational scaling factors NIST, http://srdata.nist.gov/cccbdb/vibscalejust.asp, 2017 (accessed 20.05.2017).

37. Ditchfield, J. R., Molecular Orbital Theory of Magnetic Shielding and Magnetic Susceptibility, Journal of Chemical Physics, 1972 56,5688
T.R. Sertbakan

38. Wolinski, K, Hinton, J.F., Pulay, P, Efficient implementation of the gauge-independent atomic orbital method for NMR chemical shift calculations, Journal of American Chemical Society, 1990, 112 (23), 8251-8260.

39. Azizi, N, Rostami, A.A., Godarzian, A, ${ }^{29}$ Si NMR Chemical Shift Calculation for Silicate Species by Gaussian Software, Journal of Physical Society of Japan, 2005, 74, 1609-1620.

40. Rohlfing, M, Leland, C, Allen, C, Ditchfield, R, Proton and carbon13 chemical shifts: Comparison between theory and experiment, Chemical Physics, 1984, 87, 9-15.

41. Chesnut, D, Phung, $C$, Nuclear magnetic resonance chemical shifts using optimized geometries, Journal of Chemical Physics 1989; $91,6238-6245$ 